\title{
Educação em saúde e sua contribuição no conhecimento dos usuários acerca da sífilis
}

\author{
Health education and its contribution to user knowledge about syphilis \\ La educación para la salud y su contribución al conocimiento de los usuarios sobre la sífilis
}

\begin{abstract}
RESUMO
Objetivo: Compreender o conhecimento dos usuários da estratégia de saúde da família em relação a sífilis na cidade de Cláudio-Minas Gerais. Método: Estudo qualitativo do tipo exploratório descritivo, desenvolvido em Estratégia de Saúde da Família da cidade de Cláudio - Minas Gerais, entre os meses de abril de 2018 a junho de 2018. Participaram 20 usuários. A coleta de dados foi realizada através de uma entrevista semiestruturada e gravada. Resultados: Os entrevistados possuem pouca informação relacionado a sífilis, desconhecem os sinais e sintomas, o tratamento como também os exames para diagnóstico. Em educação a saúde este tema nunca foi abordado aos entrevistados pela unidade de saúde, somente em escola e panfletos. Conclusão: Evidenciou-se que a população estudada desconhece a patologia, bem como a progressão da doença e seus meios de contágio. $O$ desconhecimento da população pode ser considerado como um ponto dificultador no que diz respeito ao controle da doença. DESCRITORES: Conhecimento; Educação em Saúde; Enfermagem; Estratégia Saúde da Família; Sífilis.
\end{abstract}

\section{ABSTRACT}

Objective: To understand the knowledge of users of the family health strategy in relation to syphilis in the city of Cláudio-Minas Gerais. Method: Qualitative descriptive exploratory study, developed in the Family Health Strategy of the city of Cláudio-Minas Gerais, between the months of April 2018 to June 2018. 20 users participated. Data collection was carried out through a semi-structured and recorded interview. Results: Respondents have little information related to syphilis, are unaware of the signs and symptoms, the treatment as well as diagnostic tests. In health education, this topic was never addressed to respondents by the health unit, only at school and pamphlets. Conclusion: It was evidenced that the studied population is unaware of the pathology, as well as the progression of the disease and its means of contagion. The population's lack of knowledge can be considered as a difficult point regarding the control of the disease.

DESCRIPTORS: Knowledge; Health education; Nursing; Family Health Strategy; Syphilis.

\section{RESUMEN}

Objetivo: Comprender el conocimiento de los usuarios de la estrategia de salud familiar en relación con la sífilis en la ciudad de Cláudio-Minas Gerais. Método: Estudio exploratorio descriptivo cualitativo, desarrollado en la Estrategia de Salud Familiar de la ciudad de Cláudio- Minas, entre los meses de abril de 2018 a junio de 2018. Participaron 20 usuarios. La recolección de datos se realizó a través de una entrevista semiestructurada y grabada. Resultados: los encuestados tienen poca información relacionada con la sífilis, desconocen los signos y síntomas, el tratamiento y las pruebas de diagnóstico. En educación para la salud, este tema nunca fue dirigido a los encuestados por la unidad de salud, solo en la escuela y los folletos. Conclusión: se evidenció que la población estudiada desconoce la patología, así como la progresión de la enfermedad y sus medios de contagio. La falta de conocimiento de la población puede considerarse como un punto difícil con respecto al control de la enfermedad.

DESCRIPTORES: Conocimiento; Educación para la salud; Enfermería; Estrategia de salud familiar; Sífilis.

RECEBIDO EM: 20/08/2020 APROVADO EM: 06/10/2020

\section{Marla Ariana Silva}

Graduanda em Enfermagem pela Universidade do Estado de Minas Gerais (UEMG), Unidade Divinópolis.

ORCID: 0000-0003-0136-7122 


\section{Karen dos Santos Lago}

Graduanda em Enfermagem pela Universidade do Estado de Minas Gerais (UEMG), Unidade Divinópolis.

ORCID: 0000-0002-4502-5098

\section{Heber Paulino Pena}

Enfermeiro. Mestre em Enfermagem. Docente do curso de Enfermagem da Universidade de Itaúna (UIT), Minas Gerais. ORCID: 0000-0002-9122-6827

\section{Fernanda Marcelino de Rezende e Silva}

Enfermeira. Doutoranda em Ciências da Saúde pela Universidade Federal de São João Del Rei (UFSJ), Campus Centro Oeste Dona Lindu (CCO). Docente e Coordenadora do Curso de Graduação em Enfermagem da Universidade do Estado de Minas Gerais (UEMG), Unidade Divinópolis.

ORCID: /0000-0003-2236-7009

\section{Silmara Nunes Andrade}

Enfermeira. Doutora em Ciências da Saúde pela Universidade Federal de São João Del Rei (UFSJ), Campus Centro Oeste Dona Lindu (CCO). Docente do Curso de Graduação em Enfermagem da Universidade do Estado de Minas Gerais (UEMG), Unidade Divinópolis.

ORCID: 0000-0002-1975-0827

\section{Regina Consolação dos Santos}

Enfermeira. Mestre em Ciência pela Universidade Federal de São João Del Rei (UFSJ), Campus Centro Oeste Dona Lindu (CCO). Docente do Curso de Graduação em Enfermagem da Universidade do Estado de Minas Gerais (UEMG), Unidade Divinópolis.

ORCID: 0000-0002-7393-3210

\section{INTRODUÇÃO}

A sífilis é uma infecção sistêmica, de evolução crônica, causada por bactéria espiroqueta, o Treponema pallidum. Doença de ampla variedade de apresentações clínicas, é conhecida desde o século XV e seu estudo ocupa todas as especialidades médicas ${ }^{(1)}$. São transmitidas principalmente pelo contato sexual ou sob forma eventual, por via sanguínea, podendo ocorrer na gestação de mãe para filho ${ }^{(2)}$.

A sífilis permanece como um problema de saúde pública mundial. A Organização Mundial de Saúde (OMS) estima que 12 milhões de pessoas sejam infectadas todos os anos. Segundo o Ministério da Saúde (MS), a taxa de detecção da sífilis aumentou de 59,1 casos por 100.000 habitantes, no ano de 2017, para 75,8 casos por 100.000 habitantes, em 2018 . Um aumento grande por se tratar de um espaço de um ano ${ }^{(1)}$.

Existem alguns fatores que podem estar correlacionados com os números elevados de casos de sífilis. Os baixos níveis de escolaridade e renda são fatores propícios ao contagio da doença, uma vez que tal público apresenta dificuldades de entendimento o que transparece a baixa escolaridade(3). Porém, estudo mostra que entre os anos de 2010 e 2017 no Brasil, houve predominância de sífilis em indivíduos com ensino médio completo ${ }^{(4)}$.

Apesar do grande número de políticas públicas, ainda há necessidade de ações preventivas que se tornem eficazes. Devido aos altos índices de casos confirmados de sífilis, torna-se importante o vínculo do profissional enfermeiro com a população a fim de tornar as ações efetivas e impactantes, na sexualidade e saúde da população. As orientações em relação a sífilis devem ser repassadas para tal público respeitando idade e os níveis de entendimento na individualidade de cada um. Para que isso aconteça de forma natural é importante e necessário que o profissional conheça o público ao qual irá atuar, o que fundamenta a pesquisa ${ }^{(5)}$.

Apesar da grande gama entre informações e campanhas realizadas acerca da sífilis, os índices de casos notificados são alarmantes, o que transparece uma lacuna nesse processo. As intervenções correlacionadas à doença devem ser efetivas para que se tenha um controle da trans-

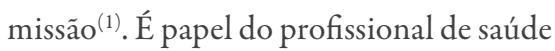
oferecer orientaçóes centrada na pessoa com vida sexual ativa e em suas práticas com o intuito de ajudá-la a reconhecer e minimizar seu risco. Diante disso, através dos altos índices da doença, percebe-se a necessidade em avaliar o conhecimento dos indivíduos em relação à sífilis, visto que a figura principal para o controle da doença é a própria população ${ }^{(3)}$.

Tais informações que dizem respeito ao conhecimento da população sobre a doença são primordiais para que os profissionais de saúde do município possam criar estratégias para sanar dúvidas da população e os principais déficits relacionados ao conhecimento acerca da doença, como por exemplo, como é realizado o tratamento e quais as principais dificuldades enfrentadas em relação a esse processo, baseando-se na amostra de indivíduos entrevistados. Diante do aumento crescente da infecção, o MS tem solicitado a ampliação do diagnóstico e tratamento da sífilis para a Atenção Primária à Saúde (APS) através da realização da testagem rápida e administração da Penicilina Benzatina conforme prescrição médica $^{(2)}$. 
Desta forma, a pesquisa utilizou de questões simples para que os participantes da pesquisa respondessem à pergunta norteadora: Qual o conhecimento dos usuários da Estratégia de Saúde da Família acerca da sífilis? Sendo assim, o artigo tem como objetivo compreender o conhecimento de usuários das estratégias de saúde da família sobre a sífilis em um município no Centro Oeste Mineiro.

\section{MÉTODOS}

Trata-se de um estudo qualitativo do tipo exploratório descritivo realizado em Estratégia de Saúde da Família (ESF) da cidade de Cláudio. O município fica localizado na região Centro Oeste de Minas Gerais, possui Índice de Desenvolvimento Humano (IDH) de 0,709 conforme o último censo (2010) e segundo estimativas do instituto Brasileiro de Geografia e Estatística (IBGE), possuía no ano de 2017 aproximadamente 28.287 habitantes ${ }^{(6)}$.

Foram selecionadas para o estudo 20 usuários de uma ESF do município que estavam na sala de espera, sendo a seleção de forma aleatória. Como critérios de inclusão, participaram do estudo homens e mulheres com faixa etária acima de 18 anos. Foram excluídos da pesquisa usuários menores de 18 anos de idade, indivíduos que recusaram em participar da pesquisa devido a desistências posteriores.

A coleta de dados foi realizada entre os meses de abril de 2018 a junho de 2018, por meio de entrevista através de questionário semiestruturado. A Entrevista semiestruturada vem se tratar de formulação de perguntas básicas para a investigação de um determinado tema, que permite ao pesquisador descobrir a realidade ${ }^{(7)}$.

Para análise, as entrevistas foram gravadas com autorização dos participantes e posteriormente transcritas. O referencial utilizado foi o de Minayo que se subdivide em etapas, sendo elas: ordenação, que se dá pela organização do material coletado, transcrição e organização das falas coletadas e classificação do material,

\section{Foram selecionadas}

para o estudo 20

usuários de uma

ESF do município

que estavam na sala

de espera, sendo a

seleção de forma

aleatória. Como

critérios de inclusão,

participaram do

estudo homens

e mulheres com

faixa etária acima

de 18 anos. Foram

excluídos da pesquisa

usuários menores

de 18 anos de

idade, indivíduos

que recusaram

em participar da

pesquisa devido

a desistências

posteriores. na qual se compara as falas coletadas com o que é realmente relevante para o trabalho, com fundamentos científicos e a análise propriamente $\operatorname{dita}^{(8,9)}$.

Os dados obtidos através do questionário foram transcritos, analisados e com base em estudos foi feita a interpretação das respostas. O material foi analisado profundamente e suas respostas deram origem a categorias e temas que responderão à pergunta norteadora.

O presente estudo obedeceu a Resolução CNS/CONEP 466 de 12 de dezembro de 2012 que aprova as diretrizes e normas de pesquisas envolvendo seres humanos ${ }^{(10)}$. Os dados somente foram coletados após a aprovação da pesquisa pelo comitê de ética em pesquisa da Universidade do Estado de Minas Gerais (COEP/ UEMG), aprovado com o número de CAAE: 89084618.3.0000.5115.

No momento da aplicação do questionário foi disponibilizado para leitura e assinatura, o Termo de Consentimento Livre e Esclarecido (TCLE), igualmente assinado, em duas vias de mesmo teor. Para manter sigilo, foi utilizado códigos para identificação dos participantes, exemplo: Usu. 1.

\section{RESULTADOS}

A partir das entrevistas foram elencadas duas categorias temáticas distintas: Conhecimentos gerais sobre a sífilis e Educação em Saúde.

\section{Conhecimentos gerais do usuário sobre a Sífilis}

Em relação aos conhecimentos básicos sobre a sífilis, podemos observar que os entrevistados possuem informações limitadas. O que nos chama a atenção é que $70 \%$ dos participantes referem saber que se trata de uma doença, porém não souberam abranger o assunto ou até mesmo explicar, outros responderam que já ouviu falar sobre o assunto, mas não se recorda mais do que se trata.

Já ouvi falar, mas não me lembro muito bem o que é (Usu. 8) 
Sei sim, é aquela doença. Não... não explicar não. Já ouvi falar (Usu. 10)

Eu sei que é uma doença, mas não sei direito como que ela é (Usu. 16) É uma doença que pegamos pelo ato sexual, é uma doença sexualmente transmissivel (Usu. 5)

Em relação a sua transmissão, $90 \%$ dos participantes responderam ser através das relaçôes sexuais, demonstrando o desconhecimento das outras formas de contágios e $10 \%$ admitiu não saber. Percebe-se que o entrevistado tem plena noção de que a sífilis se trata de uma Infecções Sexualmente Transmissíveis (IST's), é transmitida pelo ato sexual sem preservativo, porém este conhecimento entre os entrevistados encontra-se restrito.

Sei, é uma doença transmitida pela relação sexual (Usu. 9)

É uma doença que se pega através do sexo (Usu. 11)

Sim é uma doença sexualmente transmissivel (Usu. 17)

Bem, sifilis até onde eu sei é uma doença sexualmente transmissivel (Usu. 20)

Uai, sei. Se você não usar preservativo e não cuidar, acabou! (Usu. 2)

Risos: "Uai, se fizer sem (preservativo) (Usu. 6)

Não. Eu sabia, só que eu fiquei com medo de dizer (Usu. 10)

Também não (Usu. 19)

Relacionado aos sinais e sintomas da sífilis, há um desconhecimento quase que total dos entrevistados, o que é alarmante, de todos os entrevistados somente uma soube relatar alguns dos sintomas da sífilis.

Sim, verrugas na parte intima do homem e da mulher. Pode ocorrer também umas verrugas na boca e umas manchas espalhadas pelo corpo (Usu. 17)

Direcionado ao nome do exame realizado, observou-se também um desco-
Em relação a sua

transmissão, $90 \%$

dos participantes

responderam

ser através das

relações sexuais,

demonstrando o

desconhecimento

das outras formas

de contágios e $10 \%$

admitiu não saber.

Percebe-se que o

entrevistado tem

plena noção de

que a sífilis se trata

de uma Infecções

Sexualmente

Transmissíveis

(IST's), é transmitida

pelo ato sexual sem

preservativo, porém

este conhecimento

entre os entrevistados

encontra-se restrito. nhecimento por parte dos entrevistados. A minoria (20\%) dos participantes que souberam relatar o nome do exame são pessoas com um grau de escolaridade maior ou porque já chegou a realizar o exame alguma vez. A maioria respondeu ser através do exame de sangue, não sabendo especificar o nome.

Podemos observar uma insegurança ao responder as perguntas, o que transparece a dúvida e insegurança. Alguns entrevistados relataram ser através do preventivo o que é alarmante, uma vez que nos transparece o desconhecimento dos demais entrevistados. Assim deixa claro que as informações repassadas pelos profissionais não estão sendo autossuficientes, deixando uma lacuna para os usuários.
Exame de sangue? (Usu. 1)
Pelo sangue? (Usu. 2)
De sangue. Eu fiz o exame, só que eu não aprofundei em saber o que era não. Deu negativo e pronto. Vamos procurar saber só quando alguma coisa da positivo (Usu. 3)
Também não. Pelo exame de san- gue? (Usu. 5)
De sangue, não sei (Usu. 8)
Não, não é por aquele exame não? De prevenção do colo? (Usu. 4)
Não, acho que é feito junto com o preventivo (Usu. 6)
VDRL (Usu. 17)
É o VDRL, certo? (Usu. 13)

Quando perguntado sobre o conhecimento do entrevistado relacionado a alguém que já teve sífilis, somente um entrevistado relatou conhecer algum caso.
Não, não conheço (Usu. 14)
Não me lembro no momento (Usu. 16) Conheço, ela descobriu quando en- gravidou (Usu. 2)

\section{Educação em saúde}

Nessa categoria foi elencado a importância sobre a educação em saúde objetivando a prevenção de doenças por meio de ações educativas, fornecendo o empoderamento e informações relacionadas a doença. 
Houve relatos de que o exame foi realizado, mas não houve orientações relacionada a doença, outra entrevistada relatou ter visto cartazes e panfletos, porém, nenhum profissional a abordou para discutir o assunto. Na pesquisa houve uma pequena minoria que relatou ter recebido alguma orientação nas redes escolares o que demonstra que as informações repassadas aos jovens dificilmente caem no esquecimento.

Não, só realizei o exame quando fui fazer o pré-natal, mas nada explicativo. Só fiz o exame e deu negativo, nada mais foi falado (Usu. 3)

Não, ninguém me abordou ou chegou a comentar sobre isso (Usu. 8) Não, nunca foi falado (Usu. 16)

Acho que não, só vi cartazes aqui no posto (Usu. 10)

$\mathrm{Na}$ área de saúde não, mas na escola já. Depois que você começou a falar lembrei da escola (Usu. 2)

Na escola e na faculdade já, através de palestras de profissionais da saúde. Mas na unidade não (Usu. 5)

Todos os participantes negaram saber a forma de tratamento da sífilis, pois até então poucos tinham conhecimento sobre tal.

\section{DISCUSSÃO}

A partir do estudo foi possível observar que o conhecimento dos usuários da Estratégia Saúde da Família (ESF) do município de Cláudio acerca da sífilis é limitado. Grande parte das perguntas presentes no questionário não tiveram respostas corretas e/ou concretas, evidenciando um grande problema na realidade da Unidade ou até mesmo do município.

A sífilis é uma infecção sexualmente transmissível causada pela bactéria Treponema pallidum ao qual possui quatro estágios: sífilis primaria, secundária, latente e terciária. A sífilis primária se manifesta através de pequenas feridas assintomáticas, que podem aparecer na boca ou órgãos genitais. A secundária torna-se visível por meio de manchas pelo corpo, na fase latente há uma falsa impressão de melhora devido ao desaparecimento dos sintomas. Na terceira fase, o infectado apresenta alterações na pele, sistema vascular, neurológico e ossos, nesta fase o paciente pode vir a óbito. Quando diagnosticada em até um ano é a sífilis recente e após um ano da infecção esta é chamada de sífilis $\operatorname{tardia}^{(2-4)}$.

Para que haja o diagnóstico da sífilis é preciso realizar uma conjuntura entre a história do indivíduo, dados clínicos e resultados de testes laboratoriais para detecção de antígenos e anticorpos ${ }^{(4)}$. Mesmo se tratando de um exame simples no qual pode ser realizado e solicitado nas próprias unidades de saúde, o teste rápido para IST's geralmente é solicitado na maioria das vezes somente para gestantes, aliados a outros exames que compóe a rotina do pré-natal ${ }^{(2)}$. Considerando o fato de o teste rápido não ser realizado frequentemente, justifica-se a falta de conhecimento por parte dos entrevistados relacionado ao tipo de exame para detecção da sífilis.

O teste rápido para IST's é capaz de detectar dentro de 20 minutos a presença da infecção por sífilis. Ele é realizado após uma punção na polpa digital do paciente que após entrar em contato com um dispositivo portador de uma fita de nitrocelulose, acusa o resultado. Caso o paciente apresente um resultado positivo, o mesmo é encaminhado para coleta do exame laboratorial Venereal Disease Research Laboratory (VDRL) para confirmação do diagnóstico ${ }^{(11)}$.

A Educação em Saúde pode ser citada como um apanhado de ações, que tem por objetivo alterações ou acréscimos nos conhecimentos e atitudes da população, visando a melhoria da saúde e qualidade de vida e não deve ser considerada como um simples diálogo. Para que os ensinamentos se sobressaiam, é preciso que as trocas de informações sejam constantes, fazendo com que as informações repassadas não caiam no esquecimento e os mesmos possuam pensamento crítico relacionados a suas escolhas ${ }^{(12)}$.
Dos entrevistados, $70 \%$ relataram não ter recebido nenhuma informação sobre o assunto pelo profissional de saúde na ESF de referência, o que deixa claro que a Educações em Saúde não está sendo eficaz. Mesmo a sífilis sendo uma IST com tratamento simples, é notório o desconhecimento e a dúvida dos entrevistados nas respostas relatadas, o que evidência a falta de informação da população.

A falta de informação pode ser consequência de uma série de fatores que devem ser avaliados e levados em consideração. O principal deles é o conhecimento dos profissionais da área de saúde em relação a sífilis, pois a capacitação dos profissionais tem muito a acrescentar no que diz respeito a ações e abordagem da população, sendo decisórios na diminuição dos números de casos. Quanto mais informado e preparado o profissional e sua equipe estiver, melhores serão os resultados obtidos relacionados a prevenção, diagnóstico e inclusive tratamento ${ }^{(13)}$.

Dentre as atribuições do enfermeiro na Unidade Básica de Saúde podemos destacar a importância da orientação. É dever do enfermeiro orientar os indivíduos quanto a importância da utilização do preservativo, sendo essa uma dupla proteção, explicar que a multiplicidade de parceiros é considerado um fator de risco para o surgimento de possíveis infecções e enfatizar a importância da realização do exame de detecção conforme as orientações do $M S^{(14)}$.

As ações e orientações educativas tem grande influência no que se diz respeito ao controle dos altos índices de sífilis, o que faz transparecer sua importância na educação em saúde, sendo uma alternativa de prevenção. Para que esta estratégia seja válida, torna-se necessário que os profissionais da área da saúde tenham conhecimento técnico-cientifico para que sejam repassadas informações que a população realmente consiga absorver ${ }^{(15)}$.

Quanto as limitaçôes do estudo, o número da amostra de entrevistados utilizados na pesquisa é considerado uma barreira para realização do estudo, uma vez que vários usuários se mostraram resis- 


\section{artigo}

Ariana Silva, M.; Lago, K.S.; Pena, H.P.; Rezende e Silva, D.M.; Andrade, S.N.; Santos, R.C.;

Educação em saúde e sua contribuição no conhecimento dos usuários acerca da sífilis

tentes e receosos em responder algumas perguntas por se tratar de uma pesquisa sobre IST's.

\section{CONCLUSÃO}

O objetivo principal da pesquisa teve por base apurar o nível de conhecimento dos usuários da ESF. Baseando-se nas repostas dos entrevistados foi evidenciado um desconhecimento significativo em relação a patologia sífilis na ESF do município de Cláudio MG. A falta de informação pode trazer consequências negativas bem como o aumento do número de casos na cidade e região, uma vez que $70 \%$ dos entrevistados não possui conhecimentos em relação a sífilis e $100 \%$ dos participantes alegaram que este assunto nunca foi abordado na ESF.

Por fim, esse estudo faz-se necessário para reflexão dos profissionais em relação às reais informações que estão sendo repassadas para a população e se as mesmas são entendidas pelo usuário, uma vez que os níveis de entendimento são diversificados. ॠ

\section{REFERÊNCIAS}

1. Brasil. Secretaria de Vigilância em Saúde. Ministério da Saúde. Boletim Epidemiológico Sífilis, 2019 [cited 2020 ago.18]. Available from: http://www.aids.gov.br/pt-br/pub/2019/boletim-epidemiologico-sifilis-2019.

2. Brasil. Ministério da Saúde. Secretaria de Vigilância em Saúde. Departamento de Doenças de Condições Crônicas e Infecções Sexualmente Transmissíveis. Protocolo Clínico e Diretrizes Terapêuticas para Atenção Integral às Pessoas com Infecções Sexualmente Transmissíveis (IST). Brasília: Ministério da Saúde, 2020 [cited 2020 ago.18]. Available from: http://www.aids.gov. br/pt-br/pub/2015/protocolo-clinico-e-diretrizes-terapeuticas-para-atencao-integral-pessoas-com-infeccoes.

3. Brasil. Governo do Estado do Espírito Santo. Plano Estadual de Enfrentamento da Sífilis Congênita. Vitória: Espírito Santo, 2017 [cited 2020 ago.18]. Available from: https://saude.es.gov.br/ Media/sesa/Orient.\%20Cidad\%C3\%A3o/S\%C3\%ADfilis/Plano\%20 S\%C3\%ADfilis_VERS\%C3\%830\%2OFINAL.pdf.

4. Silveira SJS, Silva JQD, Damiani RF. Análise dos casos de sífilis adquirida nos anos de 2010-2017: um contexto nacional e regional. Braz. J. of Develop. 2020 [cited 2020 ago. 18]; 6(5):3249632515. Available from: https://www.brazilianjournals.com/index.php/BRJD/article/view/10862. https://doi.org/10.34117/ bjdv6n5-627.

5. Rodrigues ARM, Cavalcante AES, Silva MAM, Moreira ACA, Netto JJM, Goyanna NF. Atuação de enfermeiros no acompanhamento da sífilis na atenção primária. Rev enferm UFPE on line. 2016 [cited 2020 ago.18]; 10(4):1247-1255. Available from: https://www.researchgate.net/publication/316716885. 10.5205/reuol.8464-74011-1-SM.1004201611.

6. Instituto Brasileiro de Geografia e Estatística (IBGE). Estimativa da população, 2017 [cited 2019 dez.16]. Available from: https://www.ibge.gov.br/cidades-e-estados/mg.html.

7. Manzini EJ. Entrevista semi-estruturada: análise de objetivos e de roteiros. Seminário internacional sobre pesquisa e estudos qualitativos. 2004. [cited 2020 ago.18]. Available from: https://wp.ufpel.edu.br/consagro/files/2012/03/ MANZINI-Jos\%C3\%A9-Eduardo-Entevista-semi-estruturada-An\%C3\%A1lise-de-objetivos-e-de-roteiros.pdf.

8. Minayo MCS, Deslandes SF, Gomes R. Pesquisa social: teoria, método e criatividade. In: Pesquisa social: teoria, método e criatividade. 2016. p. 95.
9. Fontanella BJB, Ricas J, Turato ER. Amostragem por saturação em pesquisas qualitativas em saúde: contribuições teóricas. Cad. Saúde Pública [Internet]. 2008 [cited 2020 ago.18]; 24(1):17-27. Available from: https://www.scielo.br/scielo.php?pi $\mathrm{d}=s 0102-311 \times 2008000100003 \&$ script=sci_arttext. https://doi. org/10.1590/S0102-311X2008000100003.

10. Brasil. Ministério da Saúde. Conselho Nacional de Saúde. Resolução $n^{\circ}$ 466, de 12 de dezembro de 2012. Diretrizes e normas regulamentadoras de pesquisas envolvendo seres humanos. [internet]. 2012. [cited 30 jun. 2020]. Available from: https://bvsms.saude.gov.br/bvs/saudelegis/cns/2013/ res0466_12_12_2012.html

11. Silva DAR, Alves IGFG, Barros MT, Dorneles FV. Prevalência de Sífilis em mulheres. Enferm. Foco 2017 [cited 2020 ago. 18]; 8 (3): 61-64. Available from: http://revista.cofen.gov.br/index.php/ enfermagem/article/view/891/401.

12. Carmo BAG, Quadros NRP, Santos MMQ, Macena JKF, Oliveira MFV, Polaro SHI et al. Educação em saúde sobre infecções sexualmente transmissíveis para universitários de Enfermagem. Rev Bras Promoç Saúde. 2020 [cited 2020 ago.18]; 33:10285. Available from: https://periodicos.unifor.br/RBPS/article/view/10285/pdf. https:/doi. org/10.5020/18061230.2020.10285

13. Lazarini FM, Barbosa DA. Intervenção educacional na Atenção Básica para prevenção da Sífilis congênita. Rev. Latino-Am. Enfermagem [Internet]. 2017 [cited 2018 out.15];25:e2845 Available from: https://www.scielo.br/pdf/rlae/v25/pt_01041169-rlae-25-02845.pdf. https://doi.org/10.1590/15188345.1612.2845

14. Ferreira IT, Neves KTQ, Oliveira AWN, Galvão TRAF, Mangane EM, Sousa LB. Avaliação da qualidade da consulta de enfermagem em infecções sexualmente transmissiveis. Enferm. Foco. 2018 [cited 2018 out.15]; 9 (3): 42-47. Available from: http://revista.cofen.gov.br/index.php/enfermagem/article/ view/1119/459.

15. Santos SB, Machado APA, Sampaio LA, Abreu LC, Bezerra IMP. Sífilis adquirida: construção e validação de tecnologia educativa para adolescentes. J Hum Growth Dev. 2019 [cited 2018 out.15]; 29(1): 65-74. Available from: https://www.revistas.usp.br/jhgd/article/view/157752/153501. http://dx.doi. org/10.7322/jhgd.157752. 